\title{
Comparison of Clinical Features and Prognosis of Severe Fever With Thrombocytopenia Syndrome Transmitted From Tick-to-person Versus Person-to- person
}

\author{
Aiping Zhang \\ Yijishan Hospital of Wannan Medical College \\ Manman Liang \\ Yijishan Hospital of Wannan Medical College \\ Wenjie Wang \\ Yijishan Hospital of Wannan Medical College \\ Zijian Wang \\ Yijishan Hospital of Wannan Medical College

\section{Weishun Hou} \\ Yijishan Hospital of Wannan Medical College \\ Haoyu Sheng \\ Yijishan Hospital of Wannan Medical College \\ Jianghua Yang ( $\nabla$ yjhpath@163.com ) \\ Yijishan Hospital of Wannan Medical College
}

\section{Research}

Keywords: Severe fever with thrombocytopenia syndrome, Person-to-person transmission, New bunyavirus, Prognosis

Posted Date: December 18th, 2020

DOI: https://doi.org/10.21203/rs.3.rs-129900/v1

License: (c) (i) This work is licensed under a Creative Commons Attribution 4.0 International License. Read Full License 


\section{Abstract}

Background: Severe fever with thrombocytopenia syndrome (SFTS) is a tick-borne infection that has recently emerged. It is important to compare the clinical features and prognosis of severe fever with thrombocytopenia syndrome transmitted from tick-to-person versus person-to-person.

Methods: A retrospective analysis of the confirmed cases of SFTS from 2015 to 2020 in southern Anhui was performed according to the different transmission routes. The cases were divided into a tick-toperson transmission infection group (37 cases) and a person-to-person transmission infection group (12 cases).

Results: SFTS incidence was concentrated in April-June. The median time from onset to treatment was 2.5 days in the person-to-person transmission infection group and 5.0 days in the tick-to-person transmission infection group $(P<0.05)$. The proportion of consciousness disorders in the tick-to-person transmission infection group was $43.24 \%$ (16/37), which was higher than that in the person-to-person transmission infection group. The number of critical patients in the person-to-person transmission group was $16.67 \%(2 / 12)$, which was significantly lower than that in the tick-to-person transmission infection group, $91.89 \%$ (34/37). The number of deaths in the tick-to-person transmission infection group was $29.73 \%(11 / 37)$, but there were no deaths in the person-to-person transmission infection group.

Conclusions: The tick-to-person transmission infection group is more serious, and the risk of poor prognosis is higher in this group. Therefore, we should scientifically formulate prevention and control strategies and treatment plans for patients with tick infections.

\section{Introduction}

An emerging infectious disease named severe fever with thrombocytopenia syndrome (SFTS) caused by a novel phlebovirus was first reported in China in 2011[1-3]. The Haemaphysalis longicornis tick is responsible for carrying SFTS virus (SFTSV), which can spread from wildlife to people through tick bites[4]. Unexpectedly, person-to-person transmission through blood or bodily fluid has also become an important transmission route and has caused multiple cluster cases in several provinces[5-10]. Because of the high mortality and rate of person-to-person transmission, SFTS has become a serious threat to public health. The major clinical symptoms of SFTS include fever, thrombocytopenia, leukocytopenia, gastrointestinal symptoms and various other systemic manifestations, including muscular symptoms, neurological disorders and coagulopathy[11-12]. In recent years, the incidence of SFTS in Anhui Province has been increasing every year. This study retrospectively analysed the SFTS cases reported by our hospital since 2015 and provided the basis for understanding the epidemiological, clinical syndrome and symptoms and laboratory testing of SFTS cases in southern Anhui and for scientifically formulating prevention and control strategies and measures.

\section{Materials And Methods}




\section{Patients data}

A total of 49 patients who were confirmed to be previously infected by SFTSV were enrolled in this study. These patients had met one or more of the following criteria: (1) SFTSV was isolated from the patient's serum, (2) SFTSV RNA was detected in the patient's serum by quantitative reverse transcriptase polymerase chain reaction (RT-PCR) and (3) seroconversion or a 4-fold increase of antibody titres was detected in the sera between the acute and convalescent stages from the same patient. The history of epidemiology is tick-to-person or person-to-person transmission of the infection. Severe SFTS cases were defined as patients who required admission to an intensive care unit and met at least three of the following criteria[13]: (1) temperature up to $39^{\circ} \mathrm{C}$ and lasting $48-72 \mathrm{~h}$; (2) age > 60 years; (3) underlying diseases; (4) prominent neurological symptoms; (5) PLT count $<30 \times 10^{9} / \mathrm{L}$ with bleeding symptoms; (6) neutrophil (NEUT) count $\leq 1.0 \times 10^{9} / \mathrm{L}$ and WBC count $\leq 2.0 \times 10^{9} / \mathrm{L}$, as well as CK, CK-MB, and LDH levels twofold or more above the normal level.

\section{Collection of materials}

Patient data included basic information (age, sex, home address, occupation, basic disease, and time from onset to treatment, etc.), clinical symptoms (fever, fatigue, muscle soreness, abdominal pain, diarrhoea, cough/expectoration, vomiting, skin plaque/bleeding tendency, and disturbance of consciousness, etc.), haematological and blood biochemical measurements (routine blood, liver and kidney function, pancreatic function, myocardial enzyme spectrum, coagulation function, infection index, etc), and prognosis. The epidemiological data, clinical symptoms, laboratory test indexes and prognoses of the two groups were compared to understand the history of tick bite before onset, type of residence, history of outdoor activities, skin breakage, similar patient contact history, labour history, wildlife contact history, etc.

\section{Statistical analysis}

The statistical analysis was performed using SPSS software for Windows (version 20.0; IBM Corp, Armonk, NY, USA). The means for continuous variables were compared using independent-group Student's $t$ tests when the data were normally distributed; otherwise, Wilcoxon rank sum test was used. The categorical variables were compared with $\chi^{2}$ test or Fisher's exact test. A two-tailed $P<0.05$ was considered significant.

\section{Results}

\section{Epidemiological analysis}

A total of 49 eligible subjects were included in this study. They were divided into the tick-to-person transmission infection group (37 patients) and the person-to-person transmission infection group (12 patients). The incidence was concentrated in April-June. The peak of tick-to-person transmission infection was in May, accounting for $37.83 \%$ (14/37) of the cases, and the high incidence of person-to- 
person transmission infection was in April, accounting for $58.33 \%(7 / 12)$ of the cases (Fig. 1). The person-to-person transmission infection group was due to close contact with patients with SFTS, of which 8 people were exposed to blood and 4 to respiratory secretions. The following characteristics were observed in SFTS cases: a history of tick bites within 2 weeks before the onset of tick-to-person infection, living in mountainous and hilly areas, working mainly as professional farmers (81.08\%, 30/37), and participating in outdoor activities (100\%, including 18 cases of tea picking, 10 cases of mountaineering, 7 cases of mowing grass, and 2 cases of fishing).

\section{General analysis}

The median age of the person-to-person transmission infection group was 37 years old, and 8 (75\%) were male. The median age of the tick-to-person transmission infection group was 65 years old, and 14 (46.7\%) patients were male. The age difference between the two groups was statistically significant $(P=$ $0.000)$, but there was no difference in gender distribution between the two groups $(P=0.081)$. The median time from onset to treatment for the tick-to-person transmission group was significantly higher than that of the person-to-person transmission group ( 5.0 days vs 2.5 days, respectively; $P=0.002$ ). The number of severely ill patients in the person-to-person transmission group was $16.67 \%(2 / 12)$, which was significantly lower than that in the tick-to-person transmission infection group $(91.89 \%, 34 / 37: P=0.000)$. The death rate of the tick-to-person transmission infection group was significantly higher than that of the person-to-person transmission infection group ( $29.73 \%$ vs $0.0 \%$, respectively). However, there was no difference in average hospitalization days between the person-to-person transmission infection group and the tick-to-person transmission infection group ( 8.0 days vs 8.92 days, respectively; $P=0.513$ ) (Table 1). 
Table 1

Sex, age, time from onset to treatment, severe patients, hospitalization days and deaths were analyzed and compared between the person-to-person transmission infection group and tick-to-person transmission infection group.

\begin{tabular}{|c|c|c|c|c|}
\hline Characteristic & $\begin{array}{l}\text { Person-to-person } \\
\text { transmission infection } \\
\text { group }(n=12)\end{array}$ & $\begin{array}{l}\text { Tick-to-person } \\
\text { transmission infection } \\
\text { group }(n=37)\end{array}$ & Statistics & $\begin{array}{l}\mathrm{P} \\
\text { Value }\end{array}$ \\
\hline Sex, male, n (\%) & $8(75 \%)$ & $14(46.7 \%)$ & 3.044 & 0.081 \\
\hline Age, year, & $37.00(28.50,47.75)$ & $65.00(54.00,71.00)$ & -4.014 & 0.000 \\
\hline $\begin{array}{l}\text { Time from onset } \\
\text { to treatment,day }\end{array}$ & $2.50(1.00,4.75)$ & $5.00(4.00,7.00)$ & -3.111 & 0.002 \\
\hline $\begin{array}{l}\text { Severe } \\
\text { patients,n(\%) }\end{array}$ & $2(16.67 \%)$ & $34(91.89 \%)$ & 22.589 & 0.000 \\
\hline $\begin{array}{l}\text { Hospitalization } \\
\text { days }\end{array}$ & $8.0 \pm 3.72$ & $8.92 \pm 5.34$ & 0.663 & 0.513 \\
\hline Deaths,n(\%) & $0(0.00 \%)$ & $11(29.73 \%)$ & 3.051 & 0.081 \\
\hline
\end{tabular}

\section{Clinical symptom analysis}

In this study, the rate of consciousness disturbances in the tick-to-person transmission infection group was higher than that in the person-to-person transmission infection group ( $16 \mathrm{VS} 0, \mathrm{P}=0.015$ ). There was no significant difference between the two groups for other clinical symptoms (e.g., fever, fatigue, muscle soreness, cough/expectoration, abdominal pain, diarrhoea and skin plaque/bleeding tendency) $(P>0.05)$ (Table 2). 
Table 2

Clinical symptom analysis of the person-to-person transmission infection group and tick-to-person transmission infection group.

\begin{tabular}{|c|c|c|c|c|}
\hline Symptoms & $\begin{array}{l}\text { Person-to-person } \\
\text { transmission infection } \\
\text { group }(n=12)\end{array}$ & $\begin{array}{l}\text { Tick-to-person } \\
\text { transmission infection } \\
\text { group }(n=37)\end{array}$ & Statistics & $\begin{array}{l}P \\
\text { Value }\end{array}$ \\
\hline Fever, $\mathrm{n}(\%)$ & $12(100.00)$ & $37(100.00)$ & - & - \\
\hline Fatigue, $\mathrm{n}(\%)$ & $11(91.67)$ & $37(100.00)$ & - & 0.245 \\
\hline $\begin{array}{l}\text { Muscle soreness, } \\
\mathrm{n}(\%)\end{array}$ & $4(33.33)$ & 14(37.84) & 0.00 & 1.00 \\
\hline $\begin{array}{l}\text { Cough/expectoration, } \\
\mathrm{n}(\%)\end{array}$ & $1(8.33)$ & $11(29.73)$ & 1.235 & 0.266 \\
\hline Abdominal pain, $n(\%)$ & $0(0.00)$ & $7(18.92)$ & 1.329 & 0.249 \\
\hline Diarrhoea, $\mathrm{n}(\%)$ & $4(33.33)$ & 14(37.84) & 0.00 & 1.00 \\
\hline Vomiting, n(\%) & $0(0.00)$ & $13(35.14)$ & 4.078 & 0.043 \\
\hline $\begin{array}{l}\text { Skin plaque/bleeding } \\
\text { tendency, } n(\%)\end{array}$ & $0(0.00)$ & $10(27.03)$ & 2.581 & 0.108 \\
\hline $\begin{array}{l}\text { Disturbance of } \\
\text { consciousness, } n(\%)\end{array}$ & $0(0.00)$ & $16(43.24)$ & 5.864 & 0.015 \\
\hline
\end{tabular}

\section{Haematological and blood biochemical measurements analysis}

In this study, the laboratory indicators of the person-to-person transmission infection group and tick-toperson transmission infection were compared and analysed, and it was found that there were significant differences in many indexes between the two groups (Table 3 ). The routine blood indicators, including the WBC, LYM and PLT counts, were significantly lower in the tick-to-person transmission infection group than in the person-to-person transmission infection group $(P<0.05)$. The ALB level in the tick-to-person transmission infection group was lower than that in the person-to-person transmission infection group (29.81 vs 36.29 , respectively; $P<0.001$ ). There were significant differences in liver function (ALT and AST levels) between the two groups $(P<0.05)$. The GLU level in the tick-to-person transmission infection group was higher than that in the person-to-person transmission infection group ( 7.24 vs 4.58 , respectively; $\mathrm{P}=$ 0.002). The myocardial enzymatic spectrum, including CK, CK-MB, LDH, a-HBDH and TropT, were significantly higher in the tick-to-person transmission infection group than in the person-to-person transmission infection group $(P<0.05)$. There was a significant difference in the PCT level between the two groups $(P<0.05)$. The pancreatic function (AMY and LIP levels) in the tick-to-person transmission infection group was more severely affected than in the person-to-person transmission infection group ( $P$ $<0.05)$. 
Table 3

Haematological and blood biochemical measurements of the person-to-person transmission infection group and tick-to-person transmission infection group.

\begin{tabular}{|c|c|c|c|c|}
\hline $\begin{array}{l}\text { Laboratory } \\
\text { indicators }\end{array}$ & $\begin{array}{l}\text { Person-to-person } \\
\text { transmission infection group }\end{array}$ & $\begin{array}{l}\text { Tick-to-person transmission } \\
\text { infection group }\end{array}$ & Statistics & $\begin{array}{l}\mathrm{P} \\
\text { Value }\end{array}$ \\
\hline $\mathrm{WBC}\left(\times 10^{9} / \mathrm{L}\right)$ & $2.69(1.87-3.43)$ & $1.7(1.30-2.52)$ & -2.741 & 0.006 \\
\hline $\operatorname{NEUT}\left(\times 10^{9} / \mathrm{L}\right)$ & $1.00(0.78-1.35)$ & $1.00(0.70-1.35)$ & -0.217 & 0.829 \\
\hline $\operatorname{LYM}\left(\times 10^{9} / \mathrm{L}\right)$ & $0.96(0.85-2.00)$ & $0.60(0.50-0.80)$ & -2.86 & 0.004 \\
\hline $\operatorname{PLT}\left(\times 10^{9} / \mathrm{L}\right)$ & $65.00(48.00-69.75)$ & $27.00(19.50-34.00)$ & -4.351 & 0.000 \\
\hline $\operatorname{ALB}(\mathrm{g} / \mathrm{L})$ & $36.29 \pm 5.82$ & $29.81 \pm 4.40$ & -4.067 & 0.000 \\
\hline GLOB(g/L) & 24.95(20.88-29.5) & $27.50(23.83-30.78)$ & -1.371 & 0.170 \\
\hline $\operatorname{ALT}(\mathrm{U} / \mathrm{L})$ & $42.00(20.00-86.25)$ & $106.00(66.00-139.00)$ & -2.441 & 0.015 \\
\hline $\operatorname{AST}(\mathrm{U} / \mathrm{L})$ & $57.00(26.50-102.75)$ & $268.50(122.75-585.00)$ & -3.738 & 0.000 \\
\hline BUN(mmol/L) & $3.88(3.15-5.02)$ & $6.10(4.43-9.30)$ & -3.072 & 0.002 \\
\hline Cr(umol/L) & $57.65(49.75-63.50)$ & $73.75(53.58-92.28)$ & -1.488 & 0.137 \\
\hline CyS-C(mg/L) & $1.00(0.82-1.20)$ & $1.30(1.04-1.84)$ & -2.576 & 0.01 \\
\hline $\mathrm{GLU}(\mathrm{mmol} / \mathrm{L})$ & $4.58(4.43-4.85)$ & 7.24(5.33-10.47) & -3.102 & 0.002 \\
\hline $\mathrm{CK}(\mathrm{U} / \mathrm{L})$ & $255.00(100.25-687.75)$ & $683.00(333.00-1444.00)$ & -2.22 & 0.026 \\
\hline CK-MB(U/L) & $19.5 .00(18.00-25.00)$ & $47.50(33.25-72.25)$ & -3.538 & 0.000 \\
\hline $\mathrm{LDH}(\mathrm{U} / \mathrm{L})$ & $257.50(210.25-474.00)$ & $859.50(603.50-1568.75)$ & -3.762 & 0.000 \\
\hline $\mathrm{a}-\mathrm{HBDH}(\mathrm{U} / \mathrm{L})$ & $193.50(165.00-374.50)$ & $724.00(468.50-980.75)$ & -4.278 & 0.000 \\
\hline TropT(g/L) & $0.01(0.01-0.025)$ & $0.26(0.11-1.00)$ & -4.158 & 0.000 \\
\hline $\operatorname{AMY}(\mathrm{U} / \mathrm{L})$ & $94.00(48.00-150.00)$ & $199.00(149.00-315.00)$ & -3.593 & 0.000 \\
\hline $\operatorname{LIP}(\mathrm{U} / \mathrm{L})$ & $93.00(35.90-383.00)$ & $346.60(161.23-605.63)$ & -2.702 & 0.007 \\
\hline CRP(mg/L) & $7.00(4.40-10.41)$ & $11.46(4.38-26.09)$ & -2.506 & 0.182 \\
\hline РСТ(ng/ml) & $0.07(0.01-0.46)$ & $0.29(0.13-0.82)$ & -1.854 & 0.012 \\
\hline PT(s) & $11.90(11.38-12.68)$ & $12.40(11.60-13.20)$ & -1.233 & 0.217 \\
\hline APTT(s) & $33.35(31.40-37.23)$ & $58.60(46.70-67.60)$ & -3.26 & 0.001 \\
\hline
\end{tabular}

$A B L$ was compared using independent-group Student's $t$ tests. The other parameters were compared with $\chi^{2}$ test or Fisher's exact test. 


\begin{tabular}{|lllll|}
\hline $\begin{array}{l}\text { Laboratory } \\
\text { indicators }\end{array}$ & $\begin{array}{l}\text { Person-to-person } \\
\text { transmission infection group }\end{array}$ & $\begin{array}{l}\text { Tick-to-person transmission } \\
\text { infection group }\end{array}$ & Statistics & $\begin{array}{l}\mathbf{P} \\
\text { Value }\end{array}$ \\
\hline $\mathrm{TT}(\mathrm{s})$ & $20.20(18.70-22.20)$ & $26.50(22.00-41.40)$ & -2.599 & 0.01 \\
\hline $\mathrm{D}-\mathrm{D}(\mathrm{ug} / \mathrm{ml})$ & $1.39(1.03-2.09)$ & $3.88(2.04-8.12)$ & -3.011 & 0.003 \\
\hline $\begin{array}{l}\text { ABL was compared using independent-group Student's t tests. The other parameters were compared } \\
\text { with } \chi^{2} \text { test or Fisher's exact test. }\end{array}$ & & & \\
\hline
\end{tabular}

\section{Discussion}

SFTSV poses serious public health concerns globally, because it causes tick-borne haemorrhagic fever with a high case fatality rate (12-50\%)[14-15]. Currently, the pathogenic mechanism and transmission route of SFTSV are still under study. SFTS is a viral infectious disease. As a result, there is neither an effective therapeutic drug nor an effective vaccine to prevent the disease. Therefore, a retrospective analysis of the epidemiological characteristics, clinical symptoms and laboratory indicators of SFTS is of great significance for early detection, early diagnosis, early treatment and improvement of the prognosis.

According to the results of this study, SFTS incidence was concentrated in April-June. The peak of tick-toperson transmission was in May, accounting for $37.83 \%$ (14/37) of cases, and the high incidence of person-to-person transmission was in April, accounting for $58.33 \%(7 / 12)$ cases. Seasonal characteristics may be related to the decrease in the number of ticks in different regions, as may climate change, the living habits of local residents and their awareness of SFTSV. A majority of middle-aged people were found in the person-to-person transmission infection group, because health care workers are mostly middle-aged. The main route of infection was contact with the blood/respiratory secretions of a patient. Prior studies have identified direct contact with patient blood without personal protective equipment as a major risk factor for contracting SFTS[16-17]. Previous studies have suggested that non-blood contact was also associated with developing the illness[18-19]. Exposure to urine and faeces was associated with SFTSV transmission [17], and in a study by Jeong et al.[18] SFTSV RNA was detected in tracheal and gastric aspirates. Huang et al.[19] demonstrated the potential existence of infective virus particles in the mucosa and secretions of patients who may cause asymptomatic infection. The majority of the tickto-person transmission infection group were elderly farmers, a finding similar to many other studies[20]. This may be due to the local elderly engaging in tea picking, mowing and other outdoor activities (resulting in greater exposure), their own immunity status and combined with underlying diseases and so on. There was a history of tick bites in the tick-to-person transmission infection group within 2 weeks of the onset of the disease and living in mountainous and hilly areas, suggesting that SFTS has a significant regional effect[21].

SFTS clinical symptoms and laboratory tests are basically consistent with the relevant literature reports[21-22]. The clinical manifestations of the patients were mostly accompanied by fatigue, vomiting, abdominal pain, diarrhoea, muscle soreness, cough and other symptoms. Some patients had central nervous system symptoms, such as disturbance of consciousness, apathy and slow responses. In 
this study, the proportion of consciousness disorders in the tick-to-person transmission infection group was significantly higher than that in the person-to-person transmission infection group. Patients with disturbances of consciousness may develop encephalitis. A previous study reported the occurrence of encephalitis in $19.1 \%$ of patients hospitalized for SFTS, with fatal outcomes in $44.7 \%$ of these patients, based on a large sample collected over a long period[23]. For most SFTS patients, the PLT and leucocyte counts were lower than the lower limit of the normal value, with liver and kidney function damage, myocardial damage and abnormal coagulation function. This study compared the clinical laboratory indexes of the person-to-person transmission infection group to those of the tick-to-person transmission infection group and found that the differences in WBC, LYM, and PLT counts, as well as in ALB, ALT, AST, BUN, CyS-C, GLU, CK, CK-MB, LDH, a-HBDH, TropT, AMY, LIP, PCT, APTT, TT and D-D levels, were statistically significant. The results showed that the leucocyte, LYM and PLT counts decreased more obviously in the tick-to-person transmission infection group, and injuries to the myocardium, pancreas and coagulation system were more serious. LDH and CK levels and decreased LYM percentages were associated with death[24-27]. Longer APTT ( $>62.6 \mathrm{~s}$ ) has been suggested to predict a fatal outcome[28]. Moreover, the GLU level of the tick-to-person transmission infection group was higher than that of the person-to-person transmission infection group, which suggested that GLU level control in the tick-toperson infection group was poor. The reasons were as follows: (1) some patients in the tick-to-person transmission infection group had a history of diabetes mellitus; (2) the liver function of the tick-to-person transmission infection group was seriously damaged and the ability to regulate blood sugar was decreased; (3) drugs affecting blood sugar, such as glucocorticoids, were used in the tick-to-person transmission infection group. Reduced consciousness and abnormal laboratory parameters had high predictive values in identifying patients at greater risk for death. In clinical practice, these indicators could be used to reduce the fatality rate. This study also found that the number of critically ill patients in the person-to-person transmission infection group was $16.67 \%(2 / 12)$, which was significantly lower than that in the tick-to-person transmission infection group $(91.89 \%, 34 / 37)$. The number of deaths in the tickto-person transmission infection group was $29.73 \%$ (11/37), but there were no deaths in the person-toperson transmission infection group. These results suggest that tick-to-person transmission is more serious and that the risk of poor prognosis is higher for this infection group. The reason may be that most of the infected people are elderly with low immunity or more basic diseases. On the other hand, it may be caused by tick bites, which spread more viruses and/or bacteria simultaneously. Studies have shown that serum viral load levels are closely related to disease severity[29].

The investigation found that the average time from onset to treatment was 2.5 days in the person-toperson transmission infection group and 5.0 days in the tick-to-person transmission infection group, indicating that the treatment of the tick-to-person transmission infection group is not timely enough, which delays the treatment time and leads to poor prognosis[30]. The awareness of SFTSV and ticks in the high-incidence areas of Anhui Province is seriously insufficient, and the government should strengthen the propaganda and education on SFTS and ticks in high-incidence areas. Studies have shown that the interval between the onset and diagnosis is associated with mortality[31]. It is suggested that the diagnostic ability of primary medical institutions for the disease needs to be further improved, 
and training and education should be strengthened. For patients with a relevant epidemiological history and clinical manifestations, health care workers should avoid missed diagnosis.

\section{Conclusions}

Tick-to-person transmission is more serious, and the risk of a poor prognosis is higher for this infection group. This study had several limitations. First, we had no quantitative results of viral RNA. Second, the sample size was insufficient. The results will be prospectively validated by a larger cohort of patients.

\section{Abbreviations}

WBC: white blood cell; NEUT: neutrophil; LYM: lymphocyte; PLT: platelet; ALB: albumin; GLOB: globulin; ALT: alanine aminotransferase; AST: aspartate aminotransferase; BUN: blood urea nitrogen; Cr: creatinine; CyS-C: cystatin C; GLU: glucose; CK: creatine kinase; CK-MB: creatine kinase isoenzyme MB; LDH: lactate dehydrogenase; a-HBDH: a-hydroxybutyrate dehydrogenase; TropT: troponin T; AMY: amylase; LIP: lipase; CRP: C-reactive proteins; PCT: procalcitonin; PT: prothrombin time; APTT: activated partial thromboplastin time; TT: thrombin time; D-D: D-dimer; SFTS: severe fever with thrombocytopenia syndrome; SFTSV: severe fever with thrombocytopenia syndrome virus

\section{Declarations}

\section{Acknowledgments}

Not applicable.

\section{Authors' contributions}

$\mathrm{JH}$ designed the study. AP and MM analyzed the data and wrote the initial draft of the manuscript. WJ, and ZJ collected the patients data and analyzed the data. HY, JS and WS performed the statistical analysis. All authors read and approved the final manuscript.

\section{Funding}

The present study was supported by grants from The National Natural Science Foundation of China (grant nos. 30700694 and 81141083) and The Key University Science Research Project of Anhui Province, WuHu city (grant no. KJ2014A271)

\section{Availability of data and materials}

All data generated or analysed during this study are included in this published article (and its supplementary information files).

\section{Ethics approval and consent to participate}


The present study was approved by The First Affiliated Yijishan Hospital of Wannan Medical College (Wuhu, China) and it conforms to the provisions of the Declaration of Helsinki. Written informed consent was obtained from all individuals in the present study.

\section{Consent for publication}

Not applicable.

\section{Competing interests}

The authors have no conflicts of interest.

\section{References}

1. Yu XJ, Liang MF, Zhang SY, Liu L, Sun YL, Zhang L,et al. Fever with thrombocytopenia associated with a novel bunyavirus in China. N Engl J Med.2011;364:1523e32. doi.org/10.1056/NEJMoa1010095.

2. Shi J, Hu Z, Deng F, Shen S. Tick-borne viruses. Virol Sin 2018:1e23. doi.org/10.1007/s12250-0180019-0.

3. Yun SM, Lee WG, Ryou J, Yang SC, Park SW, Roh JU, et al. Severe fever with thrombocytopenia syndrome virus in ticks collected from humans,South Korea,2013. Emerg Infect Dis. 2014;20:1358. doi.org/ 10.3201/eid2008.131857.

4. Luo LM, Zhao L, Wen HL, Liu JW, Fang LZ, Xue ZF, et al. Haemaphysalis longicornis ticks as reservoir and vector of severe fever with thrombocytopenia syndrome virus in China. Emerg Infect Dis. 2015;21:1770. doi.org/10.3201/eid2110.150126.

5. Bao CJ, Guo XL, Qi X, Hu JL, Zhou MH, Varma JK, et al. A family cluster of infections by a newly recognized bunyavirus in eastern China, 2007: further evidence of person-to-person transmission. Clin Infect Dis.2011;53(12):1208-14. doi.org/10.1093/cid/cir732.

6. Gai Z, Liang M, Zhang Y, Zhang S, Jin C, Wang SW, et al. Person-to-person transmission of severe fever with thrombocytopenia syndrome bunyavirus through blood contact. Clin Infect Dis.2012;54(2):249-52. doi.org/10.1093/cid/cir776.

7. Liu Y, Li Q, Hu W, Wu J, Wang Y, Mei L, et al. Person-to-person transmission of severe fever with thrombocytopenia syndrome virus. Vector Borne Zoonotic Dis (Larchmont, NY).2012;12(2):156-60. doi.org/10.1089/vbz.2011.0758.

8. Chen H, Hu K, Zou J, Xiao J. A cluster of cases of human-to-human transmission caused by severe fever with thrombocytopenia syndrome bunyavirus. Int J Infect Dis. 2013;17(3):e206-8. doi.org/10.1016/j.ijid.2012.11.006.

9. Tang X, Wu W, Wang H, Du Y, Liu L, Kang K, et al.Human-to-human transmission of severe fever with thrombocytopenia syndrome bunyavirus through contact with infectious blood. J Infect Dis. 2013;207(5):736-9. doi.org/10.1093/infdis/jis748. 
10. Bao CJ, Qi X, Wang H. A novel bunyavirus in China. N Engl J Med. 2011; 365(9):862-3. author reply 864-5. doi.org/10.1056/NEJMc1106000.

11. Guo CT, Lu QB, Ding SJ, Hu CY, Hu JG, Wo Y, et al. Epidemiological and clinical characteristics of severe fever with thrombocytopenia syndrome (SFTS) in China: an integrated data analysis. Epidemiol Infect. 2016; 144: 1345-54. doi.org/10.1017/S0950268815002678.

12. Li DX. Severe fever with thrombocytopenia syndrome: a newly discovered emerging infectious disease. Clin Microbiol Infect. 2015; 21:614- 20. doi.org/10.1016/j.cmi.2015.03.001.

13. China National Health and Family Planning Commission. Guideline for prevention and treatment of sever fever with thrombocytopenia syndrome (2010 version). Chin J Clin Infect Dis. 2011;04(4):1934. doi.org/10.3760/cma.j.issn.1674- 2397. 2011.04.001.

14. Kim KH, Yi J, Kim G, Choi SJ, Jun KI, Kim NH, et al. Sever fever with thrombocytopenia syndrome, South Korea, 2012.Emerg Infect Dis. 2013;19: 1892-4. doi.org/10.3201/eid1911.130792.

15. Lei XY, Liu MM, Yu XJ. Sever fever with thrombocytopenia syndrome and its pathogen SFTSV. Microbes Infect. 2015;17:149-54. doi.org/10.1016/j.micinf.2014.12.002.

16. Liu K, Cui N, Fang LQ, Wang BJ, Lu QB, Peng W, et al. Epidemiologic features and environmental risk factors of severe fever with thrombocytopenia syndrome,Xinyang,China.PLoS Negl Trop Dis. 2014;8, e2820. doi.org/10.1371/journal.pntd.0002820.

17. Jiang XL, Zhang S, Jiang M, Bi ZQ, Liang MF, Ding SJ, et al. A cluster of person-to-person transmission cases caused by SFTS virus in Penglai, China. Clin Microbiol Infect. 2015;21:274e9. doi.org/10.1016/j.cmi.2014.10.006.

18. Jeong EJ, Song JY, Lim CS, Lee I, Park M-S, Choi MJ, et al.Viral shedding from diverse body ffluids in a patient with severe fever with thrombocytopenia syndrome. J Clin Virol. 2016;80:33e5. doi.org/10.1016/j.jcv.2016.04.018.

19. Huang D, Jiang Y, Liu X, Wang B, Shi J, Su Z, et al.A cluster of symptomatic and asymptomaticinfections of severe feverwith thrombocytopenia syndrome caused by person-toperson transmission. Am J Trop Med Hyg. 2017;97:396e402. doi.org/10.4269/ajtmh.17-0059.

20. Liu J, Fu H, Sun D, Wu S, Wang L, Yao M, et al. Analysis of the laboratory indexes and risk factors in 189 cases of severe fever with thrombocytopenia syndrome. Medicine.2020;99.2e18727. doi.org/10.1097/MD.0000000000018727.

21. Zhan JB, Wang Q, Cheng J, Hu B, Li J, Zhan F, et al. Current status of severe fever with thrombocytopenia syndrome in China. Virologica Sinica. 2017;32(1):51- 62. doi.org/10.1007/s12250-016-3931-1.

22. Gong L, Song DD, Wu JB, Cao MH, Su B, Sun Y, et al. Human-to-human transmissions of severe fever with thrombocytopenia syndrome virus in Anhui province, 2010-2017. Clinical Microbiology and Infection. 2018;24(8):920-2. doi.org/10.1016/j.cmi.2018.03.014.

23. Cui N, Liu R, Lu QR,Wang LY, Qin SL, Yang ZD, et al. Severe fever with thrombocytopenia syndrome bunyavirus-related human encephalitis. J Inf Secur,2015;70:52-9. doi.org/10.1016/j.jinf.2014.08.001. 
24. Zhang YZ, He YW, Dai YA, Xiong Y, Zheng H, Zhou DJ, et al. Hemorrhagic fever caused by a novel Bunyavirus in China: pathogenesis and correlates of fatal outcome. Clin Infect Dis. 2012;54:527-33. doi.org/10.1093/cid/cir804.

25. Yang ZD, Hu JG, Lu QB, Guo CT, Cui N, Peng W,et al. The prospective evaluation of viral loads in patients with severe fever with thrombocytopenia syndrome. J Clin Virol, 2016;78:123-8. doi.org/10.1016/j.jcv.2016.03.017.

26. Deng B, Zhou B, Zhang S, Zhu Y, Han L, Geng Y, et al. Clinical features and factors associated with severity and fatality among patients with severe fever with thrombocytopenia syndrome Bunyavirus infection in Northeast China. PLoS One, 2013;8:e80802. doi.org/10.1371/journal.pone.0080802.

27. Cui F, Cao HX, Wang L, Zhang SF, Ding SJ, Yu XJ, et al. Clinical and epidemiological study on severe fever with thrombocytopenia syndrome in Yiyuan County, Shandong Province, China. Am J Trop Med Hyg. 2013;88:510-2. doi.org/10.4269/ajtmh.11-0760.

28. Liu Q, He B, Huang SY, Wei F, Zhu XQ. Severe fever with thrombocytopenia syndrome,an emerging tick-borne zoonosis. Lancet Infect Dis. 2014;14:70718. doi.org/10.1016/S1473-3099(14)70718-2.

29. Li SB, Li Y, Wang QJ, Yu X, Liu M, Xie H,et al. Multiple organ involvement in severe fever with thrombocytopenia syndrome: an immunohistochemical finding in a fatal. Virology Journal .2018;15:97. doi.org/10.1186/s12985-018-1006-7.

30. Li H, Lu QB, Xing B, Zhang SF, Liu K, Du J, et al. Epidemiological and clinical features of laboratorydiagnosed severe fever with thrombocytopenia syndrome in China, 2011-17: a prospective observational study. Lancet Infect Dis,2018,18 (10) :1127-37. doi.org/10. 1016 /S1473-3099(18) 30293-7区

31. Wang L, Zou Z, Hou C, Liu X,Jiang F, Yu H. Score risk model for predicting severe fever with thrombocytopenia syndrome mortality. BMC Infect Dis. 2017;17:42. doi.org/10.1186/s12879-0162111-0.

\section{Figures}




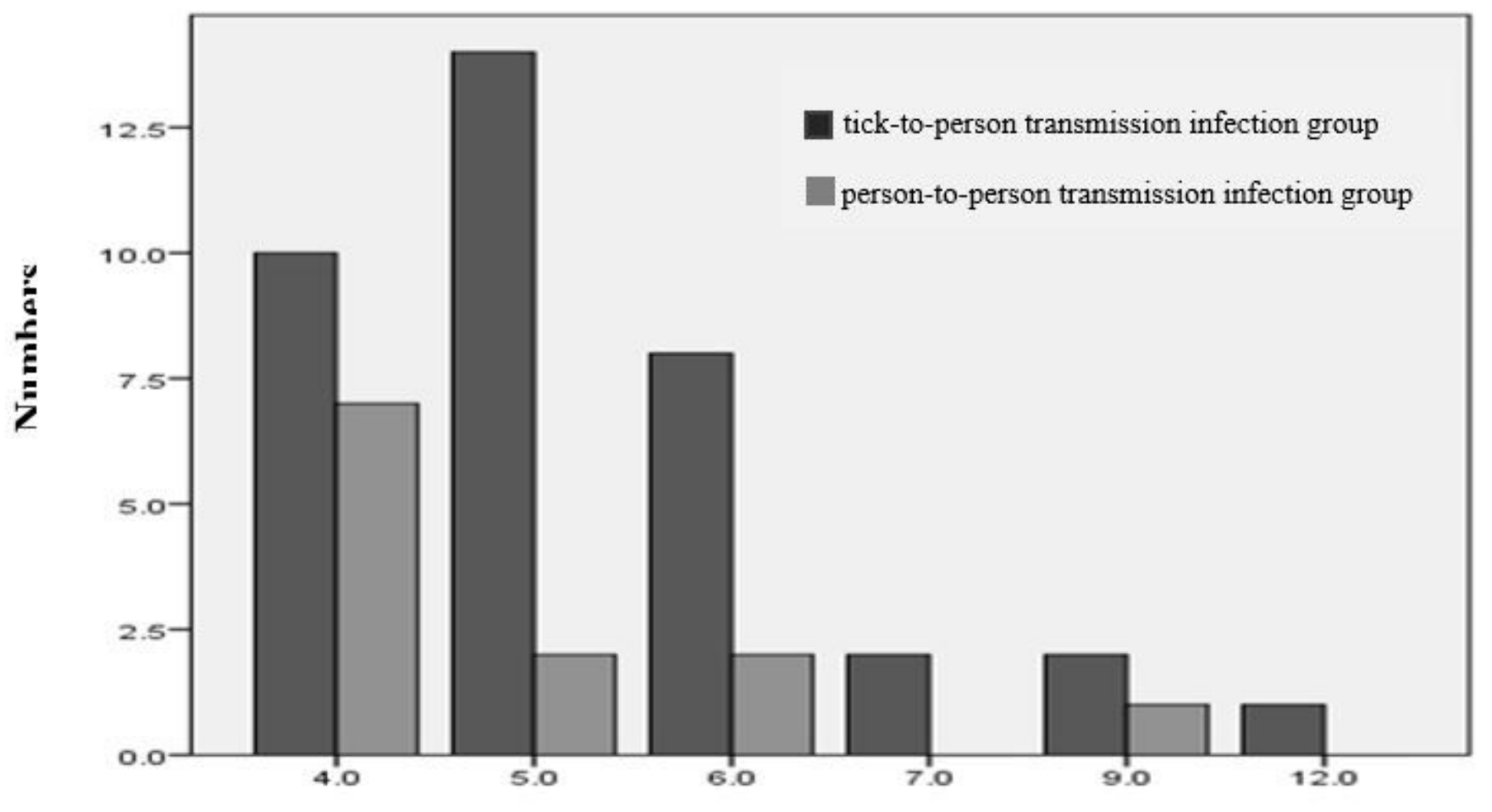

Month

Figure 1

Seasonal distribution of SFTS. The incidence of SFTS was concentrated in April-June. The peak of tickto-person transmission infection was in May, accounting for $37.83 \%(14 / 37)$ of the cases, and the high incidence of person-to-person transmission infection was in April, accounting for $58.33 \%(7 / 12)$ of the cases. 